Int. J. Dev. Biol. 50: 301-308 (2006)

doi: $10.1387 / \mathrm{ijdb} .052034 \mathrm{sp}$

\title{
Pulling forces acting on Hox gene clusters cause expression collinearity
}

\author{
SPYROS PAPAGEORGIOU* \\ Institute of Biology, NRC 'Demokritos', Aghia Paraskevi, Athens, Greece
}

\begin{abstract}
The development of normal patterns along the primary and secondary vertebrate axes depends on the regularity of early Hox gene expression. During initial stages, these expression events form a sequential pattern of partially overlapping domains along the anteroposterior axis in coincidence with the $3^{\prime}$ to $5^{\prime}$ order of the genes in the Hox cluster (spatial collinearity). In addition, the genes are activated one after the other in the 3' to 5'order (temporal collinearity). These features are poorly understood within the framework of Molecular Genetics. A model was proposed according to which physical forces act on Hox clusters as a result of signaling from morphogen gradients. The model can explain the collinearity of Hox gene expression along the primary and secondary body axes. The increase in the concentration of morphogen is accordingly followed by an increase of the force acting on the cluster. The genes are sequentially translocated, in the $3^{\prime}$ to $5^{\prime}$ order, toward the interchromosome domain where they are exposed to transcription factors for activation. The above geometrodynamic approach reproduces most collinearity data. Recent experiments verify the above prediction of sequential $3^{\prime}$ to $5^{\prime}$ Hox gene translocations in the interchromosome domain. Furthermore, it seems that these translocations, combined with cluster decondensations, are caused by attractive forces acting on the $3^{\prime}$ end of the cluster and pulling the genes out of the chromosome territory. Additional experiments are proposed in order to specify the origin of the forces.
\end{abstract}

KEY WORDS: collinearity, Hox gene expression

\section{Introduction}

In 1978, after a long series of classical genetic studies in Drosophila, E. B. Lewis established a correlation between the activation pattern of the genes of the bithorax complex $(B X-C)$ along the antero-posterior axis of the embryo and the proximodistal location of these genes along the chromosome (Lewis, 1978). This astonishing correlation (coined collinearity) proved to be a property extending to orthologous genes of all metameric animals, humans included (McGinnis and Krumlauf, 1992). It turns out that these genes contain a conserved sequence of 180 $\mathrm{bp}$, the so-called homeobox, which was first discovered in the Antennapedia and the other homeotic genes of Drosophila (McGinnis and Krumlauf, 1992; Krumlauf, 1994). Compared to Antennapedia, numerous homologue genes were found in many other genomes. These were named Hox genes and it turns out that they are grouped in complexes called HOXclusters. As in the Drosophila, Hoxgene mutations cause severe homeotic transformations and malformations of the embryonic body plan. Because of their importance in axial patterning, several homologue clus- ters have been formed by duplication in the course of evolution and their genes play significant and complementary roles in development. While Drosophila has only one homeotic complex (HOM-C), vertebrates have four such paralogous clusters Hoxa, Hoxb, Hoxc and Hoxd each one located in a different chromosome (Krumlauf, 1994). Every paralogous cluster has a variable number of genes ( 9 to 11 ) numbered from 1 to 13 in their physical order along the 3' to 5' direction on the chromosome. (In every cluster some genes of the above numbering are missing).

It was soon realized that the anteroposterior boundaries of Hox gene expression along the axis of the mouse embryo followed a collinear relationship similar to the $B X-C$ correlation established by Lewis in Drosophila (Duboule and Dollé, 1989; Graham et al., 1989). Namely, the anterior boundary of every expression was shifted along the posterior direction following the order $1,2,3, \ldots$ of the genes in the cluster (Krumlauf, 1994). This was characterized

Abbreviations used in this paper: BX-C, bithorax complex; CT, chromosome territory; ICD, interchromosome domain; np, nuclear periphery; PM, polar molecule; PPM, positive polar molecule; TF, transcription factor.

\footnotetext{
*Address correspondence to: Dr. Spyros Papageorgiou. Institute of Biology, NRC 'Demokritos', 15310 Aghia Paraskevi, Athens, Greece. Fax: +30-210-775-0001. e-mail: spapage@bio.demokritos.gr
} 
as spatial collinearity for the primary axis whereas a modified spatial collinearity was also observed for the mouse and chick limb buds (Dollé et al., 1989; Yokouchi et al., 1991) (Fig. 1). In this case, spatial collinearity takes the following form at the initial stages of activation: for the Hoxagenes at the 5 ' end of the cluster, the expression domains create a nested pattern of partially overlapping regions along the proximo-distal axis of the bud. The expression domain of the last gene, Hoxa 13, is limited at the distal tip of the bud. A similar pattern of nested expression domains is also observed for the 5' genes of Hoxdcluster with the last gene, Hoxd13, being expressed at the posterior-distal boundary of the bud (Nelson et al., 1996).

Another interesting observation in vertebrate development concerns the time dependence of Hox gene expression: the initiation of expression follows the physical order of the genes in the cluster. Gene1 is expressed first followed by gene2 etc. This sequence in time of gene activation is termed temporalcollinearity (Izpisua-Belmonte et al., 1991). A third kind of Hoxcollinearity results from the fact that the intensity at the anterior part of an

A

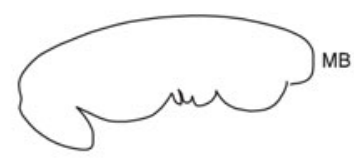

B
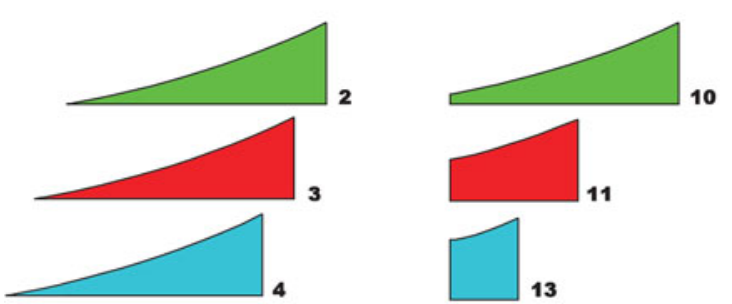

C

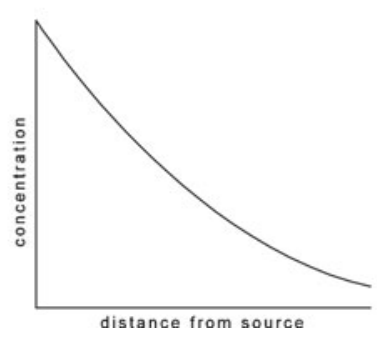

Fig. 1. Diagrams of embryos and morphogen gradients. (A) On the left, a 12 day mouse embryo is represented with the midbrain $(M B)$ to the right boundary of the embryo axis and the posterior end to the left. On the right, the limb bud is represented with the distal tip (D) to the left. (B) On the left, the schematic expression domains of Hoxb2, Hoxb3 and Hoxb4 are shown along the primary axis of the embryo. Expression is stronger anteriorly (right side) and the expression domains are shifted along the anteriorposterior axis following the order 2, 3, 4 (posterior prevalence). On the right, the expression domains of Hoxa10, Hoxa11 and Hoxa13 in the limb bud at the initial stages (around 24) are depicted. The domains form a nested pattern while distally the intensity of expression increases in the order Hoxa10, Hoxa11, Hoxa13 (quantitative collinearity). (C) A morphogen gradient with a peak at the posterior end of the vertebrate embryo or the distal tip of the limb bud. expression domain is strong compared to the posterior expression region (McGinnis and Krumlauf, 1992). In particular and in contrast to the anterior boundary, the posterior expression boundary is faint and unclear. Therefore, at every location the expression of a posterior gene is stronger and dominates over any other overlapping anterior gene (Fig. 1). This property can be related to the posterior prevalence if we assume that the protein dominance reflects the dominance in strength of its gene expression (McGinnis and Krumlauf, 1992). One can think of an equivalence between posteriorprevalenceand quantitative collinearityobserved for the 5' gene expressions of the Hoxdcluster in the limb bud (Dollé et al., 1991; Kmita etal., 2002): it turns out that distally in the bud the expression intensity systematically increases following the order Hoxd10,...,Hoxd13.

The above features of Hoxgene collinearity have been meticulously analyzed for more than 25 years in many organisms ranging from Drosophila to humans. Many attempts have been made to justify these surprising regularities but, up to now, no convincing solution has been possible based on molecular genetics and biochemical processes. Furthermore, the changes of a gene location in the cluster are associated with systematic alterations of its expression mode (Kmita et al., 2002). This leads to the hypothesis that the chromatin deforming forces in the cluster may be responsible for the expression pattern of Hox genes. I think therefore that the mechanistic nature of collinearity phenomena requires a geometrodynamical approach. I have put forward a broad framework of physical principles combined with well founded biological facts which can reasonably reproduce the observed collinearity data (Papageorgiou, 2001; Papageorgiou, 2004). The guiding dogma in this pursuit is that what happens is what can happen: phenomena that have been observed in other occasions are adopted and applied in the present formulation as long as they support the central hypothesis. This is legitimate since, in this way, the constructed model is compatible with well established facts and principles. The details of the mechanisms involved can only be determined and confirmed by experiment. It is instructive to give an example of how this has to be done. The model, in its broad formulation, proposes that collinearity is the result of physical forces translocating the Hox cluster toward a specific region where gene activation can occur. The nature, direction and application point of the forces cannot be known a priori since several possibilities can produce the expected result. After the formulation of the model an experiment has confirmed these translocations during gene activation of the Hoxb cluster (Chambeyron and Bickmore, 2004). Furthermore, this experiment has indicated where and how the force should apply on the cluster. This is helpful since it allows to write down, in the following, a detailed and more concrete version of the model. A short description is given below.

\section{A physical model for Hox gene collinearity}

The model functions at two levels in space: 1) At a multicellular ('macroscopic') level, a morphogen gradient is established over several cell diameters in morphogenetic fields of linear size approaching $1 \mathrm{~mm}$ (Wolpert, 1996; Summerbell et al., 1973) as shown in Fig. 1 for the developing chick limb bud. For many years, persistent efforts aimed to explain how these gradients are created (Green, 2002). Some recent findings corroborate a mecha- 
nism of local morphogen production and subsequent extracellular spreading and degradation of the morphogen. The spreading is diffusion-based associated with or without endocytosis depending on the specific developing system (Scholpp and Brand, 2004; Belenkaya et al., 2004). Passive diffusion may be combined with secondary procedures like morphogen transport through membranes (Kerszberg and Wolpert, 1998) or it may operate in parallel with processes like growth (Smith and Gurdon, 2004). Such secondary effects might hide the basic features of diffusion and, in order to determine the actual signal propagating mechanism, it is useful to compare the observed data with the features of signal traveling and their dependence on time and source intensity as expected in the case of pure diffusion (Papageorgiou, 1998; Vargesson et al., 2001). For simplicity here it is assumed that at the posterior end of the vertebrate embryo axis (or the distal end of the limb bud) a source starts producing a morphogen which is spread in the morphogenetic field through a mechanism based on diffusion, as described above. After a transient time interval of morphogen net production and degradation in every cell, a decreasing steady state distribution is reached of exponential form with its peak at the source area (Papageorgiou, 1998; Papageorgiou and Almirantis, 1996) (Fig. 1).

2) At the nuclear ('microscopic') level, it is assumed that the tightly packaged Hoxclusters take the shape of an elongated rigid body whose length is of the order of $500 \mathrm{~nm}$ (Papageorgiou, 2001). This is consistent with the findings that at interphase chromatin fibers are supercoiled into larger chromonema fibers whose diameters are about $80 \mathrm{~nm}$ (Belmont and Bruce, 1994; Tumbar et al., 1999) as shown in Fig. 2. These tightly clustered Hox complexes, when inactive, they are embedded inside the chromosome territory (CT) with their regulatory regions inacces-

A

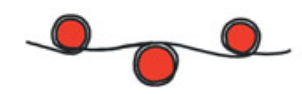

B
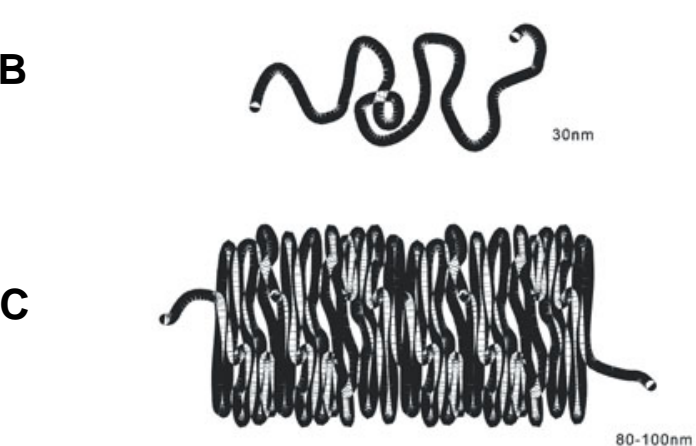

D

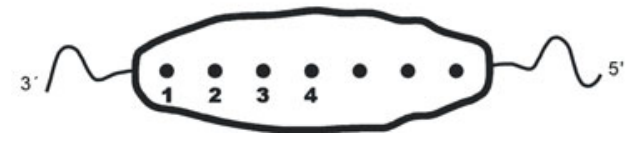

Fig. 2. Hierarchical levels of DNA packaging. (A) Nuclesomes $(11 \mathrm{~nm})$ arranged as beads-on-a-string. (B) Folded chromatin fiber of $30 \mathrm{~nm}$ diameter. (C) Condensed chromatin fiber (chromonema) of $100 \mathrm{~nm}$ diameter. (D) $A$ Hox cluster schematically shown as a rod whose length is about $500 \mathrm{~nm}$.

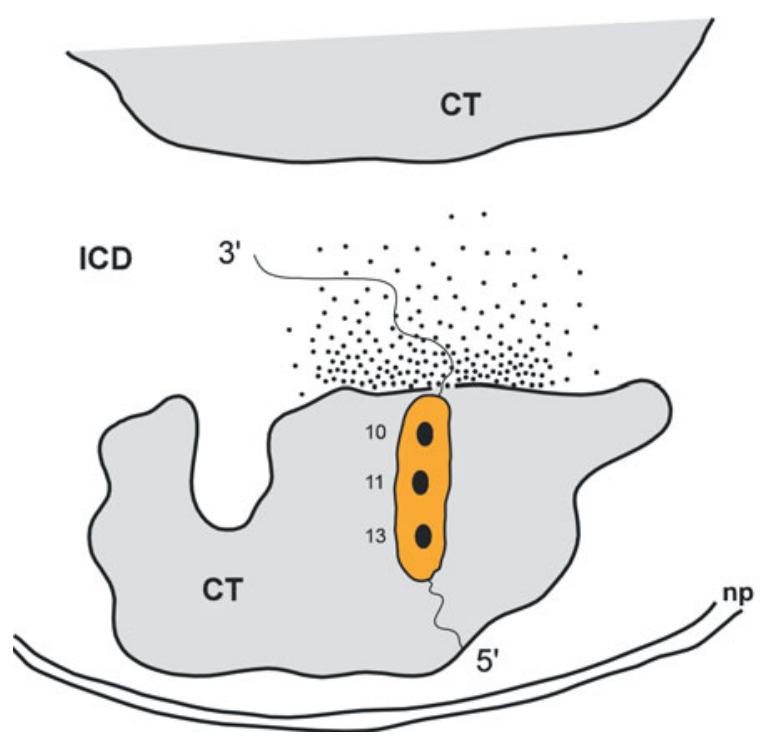

Fig. 3. Schematic drawing of a nuclear section. The single Chromosome Territory (CT) is traversed by the Interchromosome Domain (ICD) which forms a set of channels around and through the chromosome (Visseret al., 2000). Transcription factors (black spots) and other regulating molecules circulate in the ICD. Before activation, the Hox cluster is hidden inside the Chromosome Territory. At the bottom is the envelop of the nuclear periphery (np).

sible for transcription (Cremer and Cremer, 2001; Schlossherr et al., 1994). There is strong evidence that, with chromatin restructuring, gene activation occurs at the surface of the chromosome territories when genes enter in the interchromosome domain (ICD) where they can be reached by the transcription factors (Volpi et al., 2000; Cheutin et al., 2003; van Driel et al., 2003) (Figs. 3,4). The CT forms a meandering dense structure from which decondensing chromatin fibers extend (Fig. 4) (Volpi et al., 2000; Visser et al., 2000). The ICD consists of a network of channels around and through CTs. These channels are connected with nuclear pores at the nuclear surface (Visser et al., 2000). Transcription factors (TF) activating Hox genes are confined in ICD together with other mobile regulatory molecules (Cremer and Cremer, 2001). It is furthermore assumed that the density of TFs decreases away from the chromosome surface. This agrees with the observation of a concentration gradient of transcription factor Stat1 imported in the nucleus via a carrier-free (diffusion) process (Marg et al., 2004).

The morphogen-signal of the macroscopic level is transduced and uniformly amplified inside the cell so that the product molecules [PM] are produced and they act on the microscopic nuclear level. The transportation of SMAD2 inside the nucleus is an example of such a transduction and amplification (Bourillot et al., 2002; Shimizu and Gurdon, 1999). Furthermore, it was recently observed that Smad regulation in the nucleus is achieved through a physical interaction between the inner nuclear membrane MAN1 and R-Smads (Pan et al., 2005). The case of SMAD2 is not unique. Many other molecules are imported and deposited in specific locations inside the nucleus as for instance the DSH protein which is the product of Wnt signal transduction (Itoh et al., 2005). Thus, the morphogen concentration acting macroscopically is associated to a corresponding concentration of [PM] 

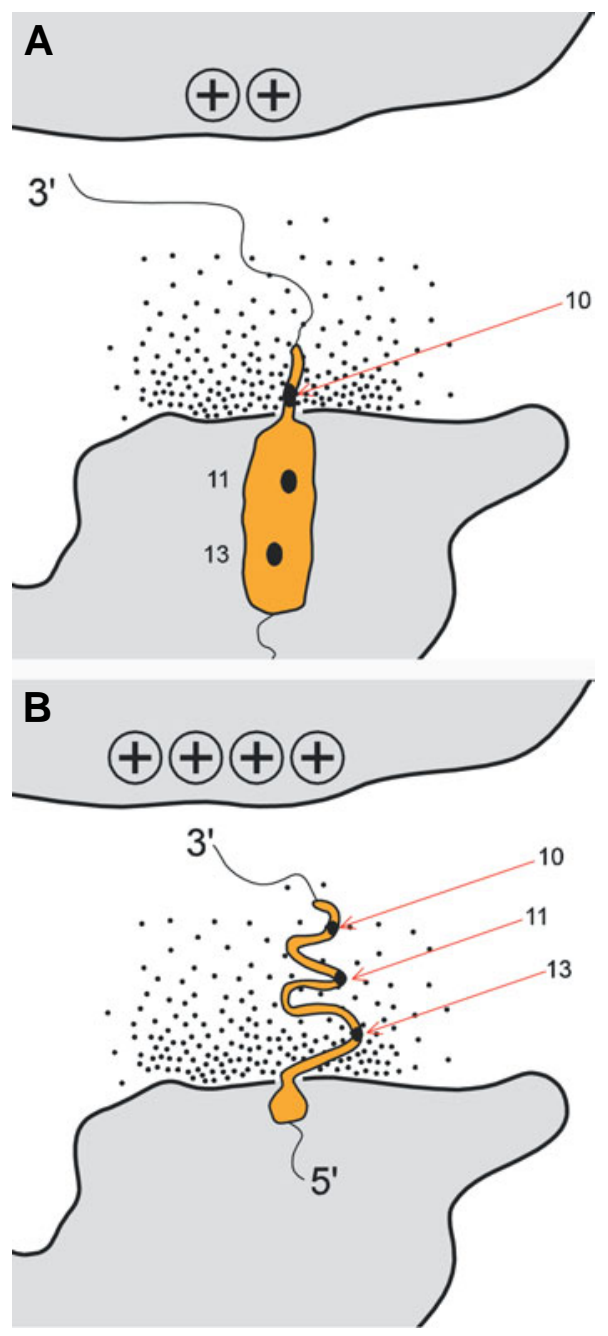

Fig. 4. Detail of Fig. 3 around the exit pore of Hoxa cluster. (A) A small electrophoretic force from the opposite Chromosome Territory surface pulls Hoxa10 out of the Chromosome Territory. (B) A stronger force stretches the fiber and pulls all three Hoxa genes inside the Interchromosomal Domain.

endowed with suitable physicochemical properties. For example, [PM] can be polar molecules that bind on the chromosome surface and collectively create an electric field (Papageorgiou, 2001; Papageorgiou, 2004). The resulting Coulomb force (attractive or repulsive) acts then on the (negatively charged) Hoxcluster (Fig. 4).

The case of electric repulsion pushing the cluster out of CT has been worked out and the existing data of spatial, temporal and quantitative collinearity have been well reproduced (Papageorgiou, 2001; Papageorgiou, 2004). Furthermore, the results of genetic deletions and duplications and the associated modifications of Hoxdexpressions in the mouse limb bud (Kmita et al., 2002) were also well described (Papageorgiou, 2004).

\section{Attractive electric forces}

Chambeyron and Bickmore have induced gene transcription of the Hoxb cluster during the differentiation of murine ES cells
(Chambeyron and Bickmore, 2004). When Hoxb1 and Hoxb9are inactive they are located inside the CT. After induction with retinoic acid, the chromatin is decondensed and an extrusion of Hoxb1 from the CT follows in association with Hoxb1 expression while Hoxb9remains inside CT. Later Hoxb9 is also shifted from inside the CT and it stays in the ICD but close to the CT surface. At the same time Hoxb1 and the decondensed chromatin fiber performs a 'choreographed looping' in ICD with an overall movement toward the centre of the nucleus (Chambeyron and Bickmore, 2004). This behaviour of Hoxb1 and Hoxb9 is in agreement with the fiber translocation according to the model.

Instead of a repulsive force applied on the 5' end of the cluster as described above, the observation of Chambeyron and Bickmore could be understood as the result of an attractive force that acts on the 3' end of Hoxbcluster and pulls the fiber toward ICD. This situation, in principle, is similar to the experiment of Cui and Bustamante who measured chromatin extensions (decondensations) as the result of pulling forces for a wide range of variation of the stretch modulus up to $25 \mathrm{pN}$ (Ciu and Bustamante, 2000). It is assumed therefore that, in response to the extracellular morphogen signals, positive polar molecules [PPM] are deposited opposite the 3' end of the cluster as shown in Fig. 4. An appropriate electric field is then acting on the negatively charged Hox cluster. The resulting electric force pulls the chromatin fiber toward the ICD and the packaged cluster is decondensed with the chromatin tethered and stretched out of the CT. Note that the same physical principles govern electrophoresis in experiments of stretching or active transport of DNA molecules and other biopolymers (Stigter and Bustamante, 1998). Electrophoretic forces are widely used to measure the elastic properties and large-scale conformational changes of chromatin fibers (Ferree and Blanch, 2003). The deformations of tethered DNA in an electric field are similar to the stretching deformations due to hydrodynamic flow (Long et al., 1996; Ferree and Blanch, 2004) and it turns out that this chromatin remodeling is necessary for DNA transcription (Katritch et al., 2000).

In cells exposed to low morphogen concentrations the resulting electrophoretic forces are weak and the Hox cluster translocations are accordingly small (Fig. 4A). A sequential increase of the morphogen concentration will respectively produce an increasing dragging force and the Hox genes will be translocated into ICD following their physical order: 1, 2, 3... (Fig. 4B). It is assumed that the density of transcription factors decreases away from the CT surface and this generates quantitative collinearity (Fig. 4). According to Fig. 4A proximally only Hoxa 10 is expressed and the intensity of expression is strong since the gene is close to the CT surface. Distally, all genes are expressed with increasing intensity (Hoxa 10, Hoxa 11, Hoxa 13) because of their relative position in ICD (Fig. 4B) in agreement with quantitative collinearity of Fig. 1B.

\section{Correlation of morphogen thresholds and gene trans- locations}

The present model can correlate morphogen thresholds with gene translocations and transcription. As an example, in Fig. 5 is shown the normal morphogen gradient $M$ which controls the activation of the 5' genes of Hoxa cluster in the limb bud. The horizontal lines $\mathrm{L}$ and $\mathrm{H}$ represent the lower and higher morpho- 


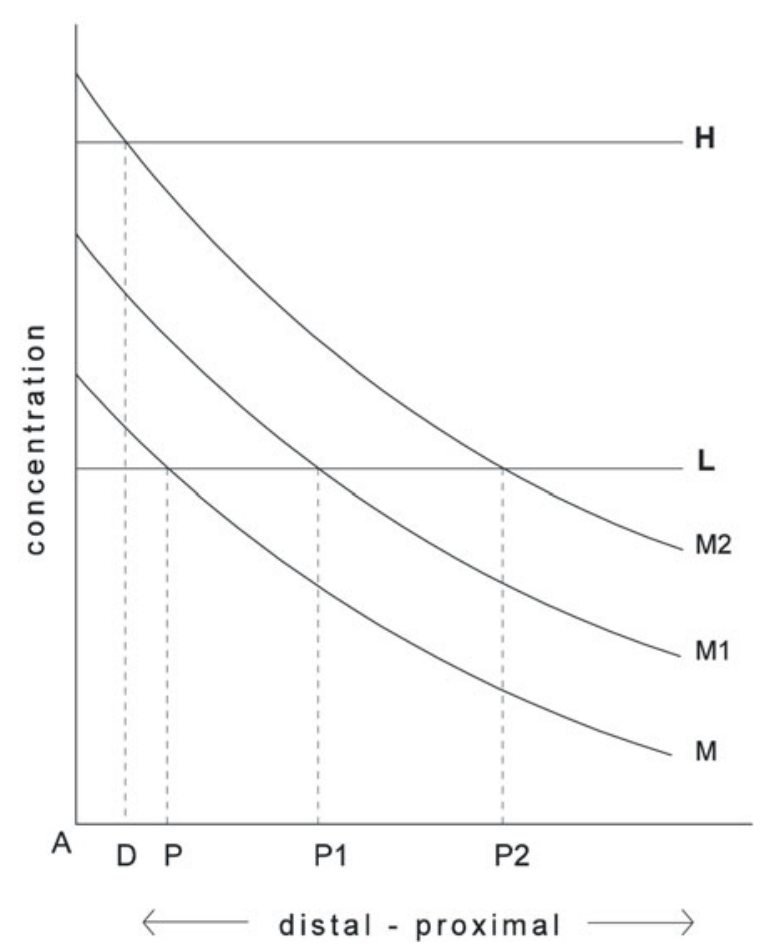

Fig. 5. Morphogens and thresholds. Normal morphogen gradient $M$ with $L$ (lower) and $H$ (higher) thresholds for Hoxa13 activation. Additional morphogen sources at the tip increase $\mathrm{M}$ to $\mathrm{M} 1$ or $\mathrm{M} 2$.

gen thresholds respectively for Hoxa13 activation in the distal region of the bud. For $M$ the expression domain of Hoxa13is (AP). When an additional morphogen source is inserted at the distal tip, the gradient increases to $M 1$ and the Hoxa13 activation domain expands to (AP1). The region (PP1) represents the domain of ectopic gene expression. If an even stronger morphogen source is inserted, the gradient increases further to M2 exceeding eventually the higher threshold $\mathrm{H}$ at the tip. In this case the ectopic expression reaches the proximal boundary $\mathrm{P} 2$ but in the region $(A D)$, where the morphogen concentration is higher than $\mathrm{H}$, the gene expression disappears.

It is instructive to interpret the above gene response to morphogen thresholds in terms of the geometry and dynamics of cluster translocations. For simplicity Hoxa13is isolated from the cluster and it is depicted in Fig. 6 for the different stages of gene activation. When Hoxa13is hidden in the interior of CT (Fig. 6A) the gene is inactive and this case corresponds to proximal cells where the morphogen level is below $\mathrm{L}$ in Fig. 5 . When the morphogen concentration increases, the associated electrophoretic force pulls the gene just above the chromosome surface (Fig. 6B). For the normal gradient $\mathrm{M}$ this occurs in proximal cells of the domain $(A P)$ in Fig. 5. When the morphogen concentration increases further, the gene is accordingly pulled stronger and it is shifted toward the border of the transcription factor region (Fig. $6 C)$. This corresponds to the distal boundary $D$ which is reached for the gradient M2 of Fig. 5. The morphogen level there is just below $\mathrm{H}$. Finally, for $\mathrm{M} 2$ the domain $(A D)$ has morphogen concentrations higher than $\mathrm{H}$, therefore the associated gene translocations are beyond the border of the transcription factor region and gene expression disappears (Fig. 6D). Such an expression attenuation has been observed for Hoxa13in the chick limb when a FGF4 bead was inserted at the distal tip of the bud (Vargesson et al., 2001). However, in this case no proximal ectopic expression was observed (PP2 in Fig. 5), as would be expected according to the model. A possible explanation of this failure is that, at st21, proximal cells in the bud are not yet competent to respond to signals for Hoxa13expression. An experiment in progress, when completed, will test the validity of this explanation (Papageorgiou, 2004).

\section{Conclusions and predictions}

The present model does not merely describe the collinearity data but its aim is to explain the origin of collinearity in terms of fundamental and well documented processes. Specific experiments have to determine the particular features of the mechanisms involved. In this spirit, different mechanisms cooperate at several levels: a) At the multicellular level and depending on the case, the study of modified diffusion with or without endocytosis has clarified how morphogen gradients can be established (Green, 2002; Scholpp and Brand, 2004; Belenkaya et al., 2004; Smith and Gurdon, 2004). b) Signal transduction has been determined with accuracy leading to the production and transportation of particular molecules in specific locations inside the nucleus (Shimizu and Gurdon, 1999; Bourillot etal., 2002; Pan etal., 2005; Itoh et al., 2005). c) At the subcellular level, physical forces are produced causing translocations of the Hoxclusters in the nucleus and as a plausible candidate an electric force (attractive or repulsive) was analyzed in more detail (Papageorgiou, 2001; Papageorgiou, 2004). The subsequent results of gene transcription in murine ES cells not only are compatible with this hypothesis but, at the same time, they specify some features of the attractive force involved (Chambeyron and Bickmore, 2004). Furthermore, some recent experiments in vivo at different stages of embryonic mouse development (Chambeyron etal., 2005) confirm the above findings and provide further support to the present model. It is still possible that, in addition to the attraction, a repulsive force may also act on the posterior end of the cluster (Papageorgiou, 2001; Papageorgiou, 2004). However, the origin of the force remains still undetermined and a direct experiment is crucial for its specification.

To the above end, a change of the electric charge of the cluster and the consequent modification of the expression pattern would be an undisputable test of the electric hypothesis. The combination of genomic rearrangement techniques (Herault et al., 1998) with the insertion methods of charged oligonucleotides in the genome (Pitard et al., 2004) could eventually serve this purpose. Accordingly, if negative charges are inserted at the 3' end of Hoxb cluster (increasing the total negative charge of the cluster) the pulling electrophoretic force will increase and the expressions of Hoxb genes will be shifted anteriorly (to the right in Fig. 1B). On the contrary, if positive charges are inserted the expressions will be shifted to the left toward the posterior end of the embryo axis.

In the present framework collinearity is the outcome of cooperation of physical principles and biochemical mechanisms. This formulation allows a unification of posterior prevalence of expressions along the embryo axis and quantitative collinearity of 5' gene expressions in the limb since, in both cases, Hox gene transcription obeys the same rules. In Fig. 5 the distance between 


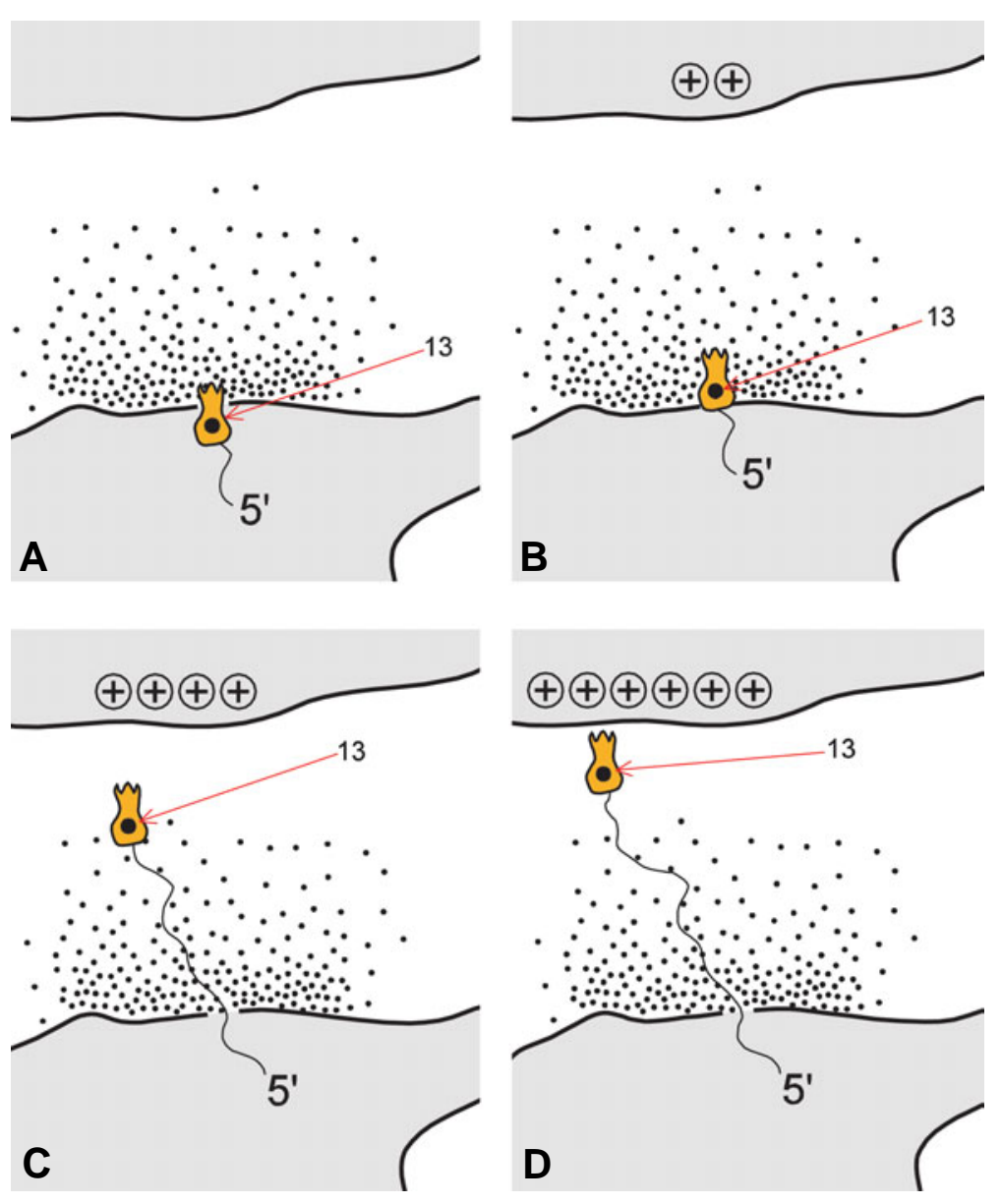

Fig. 6. Locations of Hoxa 13. (A) Inside the chromosome territory; (B) just over the surface of the chromosome territory; (C) at the outer border of a Transcription Factor area and (D) beyond the Transcription Factor area.

$\mathbf{L}$ and $\mathbf{H}$ determines a 'window between thresholds' for Hoxa13 gene expression. This is associated to the fiber stretching inside the TF area (Fig. 4) and corresponds to a 'window of gene expression' contained between the spatial boundaries (proximal and distal). In this respect, the difference between partially overlapping and nested expression domains is only accidental: the 3' genes in the primary embryo axis cannot be accommodated in the TF area and for every cell only a subset of these gene expressions fits in. In the limb bud, when extruded in the ICD, the 5' genes can be contained in the TF area and they form a nested pattern. In both cases the posterior gene expression dominates compared to the effect of the overlapping more anterior gene expressions. Furthermore, an FGF4 bead inserted distally in the bud will increase the morphogen level above $\mathbf{H}$ at the tip area (Fig. 5) and accordingly Hoxa13 gene will be shifted beyond the window of gene expressions (Fig. 6). The verification of the gradual Hoxa13 attenuation distally (Vargesson, 2001) indicates that, as in the primary axis, the nested expression pattern can become partially overlapping as a result of appropriate gene translocations.

If the electric hypothesis proves correct, many specific questions could be asked: how reversible is the decondensed Hoxfiber toward the initial condensed state inside the CT when the gene activation is turned off? In this spirit, a regression of Hoxb1 was noticed from the ICD toward the CT at later stages of activation (Chambeyron and Bickmore, 2004).

There are some experiments where the collinearity picture is violated. For instance when the anterior Hoxb1 is ectopically relocated at the posterior end of the HoxD cluster near Hoxd13 the following expressions were observed (Kmita et al., 2000): normally Hoxb1 is expressed in the fourth rhombomere of the developing nervous system. The expression of the relocated Hoxb1 transgene is totally abolished in agreement with the present model since the posterior Hoxd genes are not expressed in this rhombomere. However the transgene expression in the mesoderm was unexpectedly not suppressed. This probably indicates that the normal collinearity behaviour depends on the developing stage, the tissue and the transient structure of the chromatin architecture. Thus, it seems that in the early mesoderm the chromatin is not dense enough and the factors of Hoxb1 activation can recognize the ectopic transgene inducing a local opening in the neighborhood of Hoxd13(Kmita et al., 2000). In the same article the authors describe some specific mechanisms to this end (Kmita et al., 2000).

In Drosophila the developmental fate of the domains along the anterior-posterior axis is specified by the repression of the homeotic gene expressions (Busturia and Bienz, 1993). Apart from the obvious differences from the present formulation, the ABD-B protein spreads across several parasegments in the posterior- anterior direction in a manner 'vaguely reminiscent' of the spreading of the posterior Hox genes (Abd-B-like genes) in the developing vertebrate limb (Dollé et al., 1989; Busturia and Bienz, 1993).

Physical forces are involved in many developmental processes (Gordon, 1999). In the case of Hox gene collinearity I think it is impossible to explain the observed regularities without using a physical principle, for the following reason: as exposed above, the chemical (molecular) cue of positional information is transformed into a chemical signal at the chromatin level. One biochemical response to this signaling is the high increase in histone $\mathrm{H} 3$ acetylation and methylation (Chambeyron and Bickmore, 2004). However, these histone modifications are not sufficient to produce the HoxB cluster decondensation which is the necessary first step for gene activation (Chambeyron and Bickmore, 2004). Only physical forces can cause such mechanical modifications of large molecular complexes (e.g. decondensation, stretching, translocation, shearing etc) and the observed collinearity reflects the correlation between the measure of the applied force and the degree of chromatin (cluster) deformation. The explanation of collinearity therefore should be based on appropriate physical mechanisms generating suitable forces acting on the nuclear macromolecules. Gene enhancers, inhibitors, cis-regulators and the other tools of the biochemical machinery, although crucial, cannot explain only by themselves all aspects of collinearity (Papageorgiou, 2004). This inability and the accumulation of new complicated data led to the assumption that no single and universal mechanistic explanation may exist for these correlated phenomena (Kmita and Duboule, 2003). Instead, tinkering without underlying logic other than the attainment of the final goal might be responsible for the observed 
characteristic gene expressions. In contrast, according to the present formulation, collinearity is unique and universal since it is the result of physical laws. However, in every patterning pathway along axes, the backbone of collinearity is covered by the features of the particular geometry (macroscopic and microscopic) combined with the molecular specificity of the by-side genetic activation. For example, collinearity in the primary vertebrate axis generates partially overlapping expressions while in the limb bud the expressions form a nested pattern (Fig. 1B). In other cases, the chromosome environment of the cluster does not fulfill the prerequisites for the proper appearance of Hox gene collinearity (Kmita et al., 2000). Whenever the universal phenomenon of collinearity is at work, the phenotypes in each case are modulated by geometry and the concurrent molecular and genetic processes. Future experiments will clarify whether tinkering or the 'dressed' core of collinearity is responsible for the observed variety of axial developmental events.

\section{References}

BELENKAYA, T.Y., HAN, C., YAN, D., OPOKA, R.J., KHODOUN, M., LIU, H. and LIN, X. (2004). Drosophila Dpp morphogen movement is independent of dynamin-mediated endocytosis but regulated by the glypican members of heparan sulfate proteoglycans. Cel/119:231-244.

BELMONT, A.S. and BRUCE, K. (1994). Visualization of G1 chromosomes: a folded, twisted, supercoiled chromonema model of interphase chromatid structure. J Cell Biol. 127:287-302.

BOURILLOT, P.Y., GARRETT, N. and GURDON, J.B. (2002). A changing morphogen gradient is interpreted by continuous transduction flow. Development 129:2167-2180.

BUSTURIA, A. and BIENZ, M. (1993). Silencers in Abdominal-B, a homeotic Drosophila gene. EMBO J. 12:1415-1425.

CHAMBEYRON, S. and BICKMORE, W.A. (2004). Chromatin decondensation and nuclear reorganization of the HoxBlocus upon induction of transcription. Genes \& Dev. 18:1119-1130.

CHAMBEYRON, S., DA SILVA, N.R., LAWSON, K.A. and BICKMORE, W.A. (2005). Nuclear re-organisation of the Hoxb complex during mouse embryonic development. Development 132:2215-2223.

CHEUTIN, Y., MCNAIRN, A.J., JENUWEIN, T., GILBERT, D.M., SINGH, P.B. and MISTELI, T. (2003). Maintenance of stable heterochromatin domains by dynamic HP1 binding. Science 299:721-725.

CREMER, T. and CREMER, C. (2001). Chromosome territories, nuclear architecture and gene regulation in mammalian cells. Nat. Rev. Genet. 2: 292-301.

CUI, Y. and BUSTAMANTE, C. (2000). Pulling a single chromatin fiber reveals the forces that maintain its higher-order structure. Proc. Natl. Acad. Sci. USA 97:127-132.

DOLLÉ, P., IZPISUA-BELMONTE, J.-C., FALKENSTEIN, H., RENUCCI, A. and DUBOULE, D (1989). Coordinate expression of the murine Hox-5 complex homeobox-containing genes during limb pattern formation. Nature 342:767772.

DOLLÉ, P., IZPISUA-BELMONTE, J.-C., BROWN, J.M., TICKLE, C. and DUBOULE, D. (1991). HOX-4 genes and the morphogenesis of mammalian genitalia. Genes \& Dev 5:1767-1777.

DUBOULE, D. and DOLLÉ, P. (1989). The structural and functional organization of the murine HOX gene family resembles that of Drosophila homeotic genes. EMBO J. 8:1497-1505.

FERREE, S. and BLANCH, H.W. (2003). Electrokinetic stretching of tethered DNA. Biophys. J. 85:2539-2546.

FERREE, S. and BLANCH, H.W. (2004). The hydrodynamics of DNA electrophoretic stretching and relaxation in a polymer solution. Biophys. J. 87:468-475.

GORDON, R. (1999). The Hierarchical Genome and Differentiation Waves. London: World Scientific.

GRAHAM, A., PAPALOPULOU, N. and KRUMLAUF, R. (1989). The murine and
Drosophila homeobox gene complexes have common features of organization and expression. Cel/57:367-378.

GREEN, J. (2002). Morphogen gradients, positional information and Xenopous: interplay of theory and experiment. Dev. Dyn. 225:392-408.

HERAULT, Y., RASSOULZADEGAN, M., CUZIN, F. and DUBOULE, D. (1998). Engineering chromosomes in mice through targeted meiotic recombination (TAMERE). Nat. Genet. 20:381-384.

ITOH, K., BROTT, B.K., RATCLIFFE, M.J. and SOKOL, S.Y. (2005). Nuclear localization is required for Dishevelled function in Wnt/beta-catenin signalling. J. Biol. 4(1):3.

IZPISUA-BELMONTE, J.-C., FALKENSTEIN, H., DOLLÉ, P., RENUCCI, A. and DUBOULE, D. (1991). Murine genes related to the Drosophila $A b d B$ homeotic gene are sequentially expressed during development of the posterior part of the body. EMBO J. 10:2279-2289.

KATRITCH, V., BUSTAMANTE, C. and OLSON, W.K. (2000). Pulling chromatin fibers: computer simulations of direct physical micromanipulations. J. Mol. Biol. 295:29-40.

KERSZBERG, M. and WOLPERT, L. (1998). Mechanisms for signaling by morphogen transport: a theoretical study. J. Theor. Biol. 191:103-114.

KMITA, M., VAN DER HOEVEN, F., ZAKANY, J., KRUMLAUF, R. and DUBOULE. D. (2000). Mechanisms of Hox gene colinearity: transposition of the anterior Hoxb1 gene into the posterior HoxD complex. Genes \& Dev. 14:198-211.

KMITA, M., FRAUDEAU, N., HÉRAULT, Y. and DUBOULE, D. (2002). Serial deletions and duplications suggest a mechanism for the collinearity of Hoxd genes in limbs. Nature 420:145-150.

KMITA, M. and DUBOULE, D. (2003). Organizing axes in time and space; 25 years of colinear tinkering. Science 301:331-333.

KRUMLAUF, R. (1994). Hox Genes in Vertebrate Development. Cel/78:191-201.

LEWIS, E.B. (1978). A gene complex controlling segmentation in Drosophila. Nature 276:565-570.

LONG, D., VIOVY, J. and AJDARI, A. (1996). Stretching DNA with electric fields revisited. Biopolymers 39: 755-759.

MARG, A., SHAN, Y., MEYER, T., MEISSNER, T., BRANDENBURG, M. and VINKEMEIER, U. (2004). Nucleocytoplasmic shuttling by nucleoporins Nup153 and Nup214 and CRM1-dependent nuclear export control the subcellular distribution of latent Stat1. J. Cell Biol. 165:823-833.

MCGINNIS, W. and KRUMLAUF, R. (1992). Homeobox Genes and Axial Patterning. Cel/68:283-302.

NELSON, C.E., MORGAN, B.A., BURKE, A.C., LAUFER, E., DIMAMBRO, E., MURTAGH, L.C., GONZALEZ, E., TESSAROLLO, L., PARADA, L.F. and TABIN, C. (1996). Analysis of Hox gene expression in the chick limb bud. Development 122:1449-1466.

PAN, D., ESTEVEZ-SALMERON, L.D., STROSCHEIN, S.L., ZHU, X., HE, J., ZHOU, S. and LUO, K. (2005). The integral inner nuclear membrane protein MAN1 physically interacts with the R-Smad proteins to repress signalling by the transforming growth factor-\{beta\} superfamily of cytokines. J. Biol. Chem. 280:15992-16001.

PAPAGEORGIOU, S. (1998). Cooperating morphogens control hoxdgene expression in the developing vertebrate limb. J. Theor. Biol. 192:43-53.

PAPAGEORGIOU, S. (2001). A physical force may expose Hox genes to express in a morphogenetic density gradient. Bull. Math. Biol. 63:185-200.

PAPAGEORGIOU, S. (2004). A cluster translocation model may explain the collinearity of Hox gene expressions. BioEssays 26:189-195.

PAPAGEORGIOU, S. and ALMIRANTIS, Y. (1996). Gradient model describes the spatial-temporal expression pattern of Hoxagenes in the developing vertebrate limb. Dev. Dyn. 207:461-469.

PITARD, B., BELLO-ROUFAI, M., LAMBERT, O., RICHARD, P., DESIGAUX, L., FERNANDES, S., LANCTIN, C., POLLARD, H., ZEGHAL, M., RESCAN, P.Y. and ESCANDE, D. (2004). Negatively charged self-assembling DNA/poloxamine nanospheres for in vivo gene transfer. Nucleic Acids Res. 32(20):e159.

SCHLOSSHERR, J., EGGERT, H., PARO, R., CREMER, S. and JACK, R.S. (1994). Gene inactivation in Drosophila mediated by polycomb gene product or by position-effect variegation does not involve major changes in the accessibility of the chromatin fibre. Mol. Gen. Genet. 243:453-462.

SCHOLPP, S. and BRAND, M. (2004). Endocytosis controls spreading and effec- 


\section{S. Papageorgiou}

tive signaling range of Fgf8 protein. Curr. Biol. 14:1834-1841.

SHIMIZU, K. and GURDON, J.B.(1999). A quantitative analysis of signal transduction from activin receptor to nucleus and its relevance to morphogen gradient interpretation. Proc. Natl. Acad. Sci. USA 96:6791-6796.

SMITH, J.C. and GURDON, J.B. (2004). Many ways to make a gradient. BioEssays 26:705-706.

STIGTER, D. and BUSTAMANTE, C. (1998). Theory for the hydrodynamic and electrophoretic stretch of tethered B-DNA. Biophys. J. 57:1197-1210.

SUMMERBELL, D., LEWIS, J.H. and WOLPERT, L. (1973). Positional information in chick limb morphogenesis. Nature 244:492-496.

TUMBAR, T., SUDLOW, G. and BELMONT, A.S. (1999). Large-scale chromatin unfolding and remodeling induced by VP16 acid activation domain. J. Cell Biol. 145:1341-1354.

VAN DRIEL, R., FRANSZ, P.F. and VERSCHURE, P.J. (2003). The eukaryotic genome: a system regulated at different hierarchical levels. J. Cel/Sci. 116:40674075 .
VARGESSON, N., KOSTAKOPOULOU, K., DROSSOPOULOU, G., PAPAGEORGIOU, S. and TICKLE, C. (2001). Characterization of Hoxagene expression in the chick limb bud in response to FGF. Dev. Dyn. 220:87-90.

VISSER, A.E., JAUNIN, F., FAKAN, S. and ATEN, J.A. (2000). High resolution analysis of interphase chromosome domains. J. Cell SCi. 113:2585-2593.

VOLPI, E.V., CHEVRET, E., JONES, T., VATCHEVA, R., WILLIAMSON, J., BECK, S., CAMPBELL, R.D., GOLDWORTHY, M., POWIS, S.H. and RAGOUSSIS, J. (2000). Large-scale chromatin organization of the major histocompatibility complex and other regions of human chromosome 6 and its response to interferon in interphase nuclei. J. Cell Sci. 113:1565-1576.

WOLPERT, L. (1996). One hundred years of positional information. Trends Genet. 12:359-364.

YOKOUCHI, Y., SASAKI, H. and KUROIWA, A. (1991). Homeobox gene expression correlated with the bifurcation process of limb cartilage development. Nature 353:443-445. 